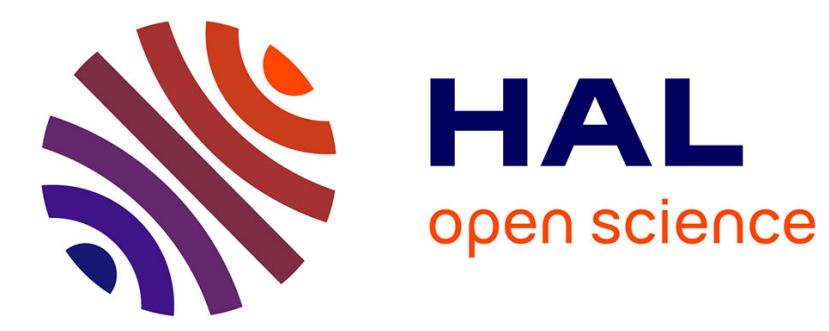

\title{
Les études cas-non cas : principe, méthodes, biais et interprétations
}

\author{
Jean-Luc Faillie
}

\section{To cite this version:}

Jean-Luc Faillie. Les études cas-non cas : principe, méthodes, biais et interprétations. Thérapie, 2018, 73 (3), pp.247-255. 10.1016/j.therap.2017.08.006 . hal-01741153

\section{HAL Id: hal-01741153 \\ https://hal.science/hal-01741153}

Submitted on 2 Oct 2019

HAL is a multi-disciplinary open access archive for the deposit and dissemination of scientific research documents, whether they are published or not. The documents may come from teaching and research institutions in France or abroad, or from public or private research centers.
L'archive ouverte pluridisciplinaire HAL, est destinée au dépôt et à la diffusion de documents scientifiques de niveau recherche, publiés ou non, émanant des établissements d'enseignement et de recherche français ou étrangers, des laboratoires publics ou privés. 


\title{
Les études cas-non cas : principe, méthodes, biais et interprétations ${ }^{\text {th }}$
}

\section{Case-non case studies: Principles, methods, bias and interpretation}

\author{
Jean-Luc Faillie ${ }^{a, *, b}$
}

\begin{abstract}
a Département de pharmacologie médicale et toxicologie, centre régional de pharmacovigilance, CHU de Montpellier, 371, avenue du doyen Gaston-Giraud, 34295 Montpellier, France

${ }^{\mathrm{b}}$ EA2415, laboratoire de biostatistiques, épidémiologie et santé publique, faculté de médecine, institut universitaire de recherche clinique, université de Montpellier, 34295 Montpellier, France
\end{abstract}

Reçu le 20 juin 2017 ; accepté le 31 août 2017

Disponible sur Internet le 31 octobre 2017

\section{MOTS CLÉS}

Étude cas-non cas ; Analyse de disproportionnalité ; Reporting odds ratio ;

Pharmacovigilance ; Pharmacoépidémiologie ; Biais

\begin{abstract}
Summary Case-non case studies belongs to the methods assessing drug safety by analyzing the disproportionality of notifications of adverse drug reactions in pharmacovigilance databases. Used for the first time in the 1980s, the last few decades have seen a significant increase in the use of this design. The principle of the case-non case study is to compare drug exposure in cases of a studied adverse reaction with that of cases of other reported adverse reactions and called "non cases". Results are presented in the form of a reporting odds ratio (ROR), the interpretation of which makes it possible to identify drug safety signals. This article describes the principle of the case-non case study, the method of calculating the ROR and its confidence interval, the different modalities of analysis and how to interpret its results with regard to the advantages and limitations of this design.
\end{abstract}

(C) 2017 Société française de pharmacologie et de thérapeutique. Published by Elsevier Masson SAS. All rights reserved.

\footnotetext{
it Cet article a fait l'objet d'une présentation par l'auteur lors du $3^{\mathrm{e}}$ séminaire de pharmaco-épidémiologie des Centres régionaux de pharmacovigilance (CRPV) \& Centres d'évaluation et d'information sur la pharmacodépendance-addictovigilance (CEIP), organisé par le Réseau français des CRPV (RCRPV) à Tours, les 1-2 juin 2017.

* Correspondance.

Adresse e-mail : jl-faillie@chu-montpellier.fr
} 


\section{KEYWORDS}

Case-non case study; Disproportionality analysis;

Reporting odds ratio; Pharmacovigilance; Pharmacoepidemiology; Bias
Résumé Les études de type cas-non cas appartiennent aux méthodes ayant pour objectif d'évaluer la sécurité d'emploi des médicaments en analysant la disproportionnalité des notifications d'effets indésirables médicamenteux dans les bases de données de pharmacovigilance. Utilisé pour la première fois dans les années 1980, les dernières décennies ont vu une augmentation importante de l'utilisation de ce design d'étude. Le principe des études cas-non cas réside dans la comparaison de l'exposition médicamenteuse de cas d'un effet indésirable d'intérêt avec celle de cas ayant présenté d'autres effets et appelés «non cas ». Leurs résultats sont présentés sous la forme de reporting odds ratio (ROR) dont l'interprétation permet de mettre en évidence des signaux de pharmacovigilance. Cet article décrit le principe des études cas-non cas, la méthode de calcul du ROR et de son intervalle de confiance, les différentes modalités d'analyse et présente comment interpréter ses résultats au regard des avantages et des limites de ce type d'étude.

(C) 2017 Société française de pharmacologie et de thérapeutique. Publié par Elsevier Masson SAS. Tous droits réservés.

\section{Abréviations}

AERS adverse event reporting system

ANSM Agence nationale de sécurité du médicament et des produits de santé

ATC (classification) anatomique, thérapeutique et chimique

AVC accident vasculaire cérébral

BCPNN Baysian confidence propagation neural network

EMA Agence européenne du médicament

FDA Food and drug administration

IEC inhibiteur de l'enzyme de conversion

HERG human ether-a-go-go related gene

MGPS multi item gamma Poisson Shrinker (method)

OMS Organisation mondiale de la santé

ROR reporting odds ratio

PRR proportional reporting ratio

SMQ standardised MedRA queries

THS traitements hormonaux substitutifs

\section{Introduction}

Les études cas-non cas appartiennent aux études dites de disproportionnalité qui concernent spécifiquement l'analyse des bases de données de notifications spontanées d'effets indésirables médicamenteux. Ces bases de données recensent les cas d'effets indésirables notifiés au système de pharmacovigilance au niveau national, comme la base de données nationale de pharmacovigilance française gérée par l'Agence nationale de sécurité du médicament et des produits de santé (ANSM) ou l'adverse event reporting system (AERS) de la Food and drug administration (FDA) aux ÉtatsUnis, ou au niveau international, comme la base de données européenne EudraVigilance (gérée par l'Agence européenne du médicament [EMA]), ou encore la base de données de l'Organisation mondiale de la santé (OMS), VigiBase.

L'étude de la disproportionnalité des notifications spontanées a pour objectif de générer le plus précocement possible après la commercialisation des signaux de pharmacovigilance concernant des effets indésirables médicamenteux non connus ou sous-estimés. Dans ce contexte, on entend par signal, un nombre de notifications de l'effet indésirable d'intérêt supérieur à celui attendu, c'est-à-dire un taux de notification « disproportionné » d'un effet indésirable particulier associé à un médicament d'intérêt par rapport aux autres effets notifiés dans une base de données de pharmacovigilance. Les études de disproportionnalité sont utilisées soit lors de la détection automatisée de signaux dans une base de données soit pour investiguer une hypothèse issue de l'analyse pharmacologique ou de l'analyse de données observées chez des patients exposés et faisant suspecter un risque médicamenteux. Les études de disproportionnalité connaissent une utilisation croissante depuis les années 1990. En effet, le développement et la disponibilité des bases de données de pharmacovigilance ont permis la réalisation de ce type d'étude par les pharmacovigilants, d'autant plus qu'elle ne nécessite pas d'importants moyens sur le plan logistique. Parallèlement, les fréquentes crises sanitaires concernant la sécurité d'emploi des médicaments et l'absence de réelle amélioration de l'évaluation des risques avant la commercialisation des médicaments renforcent la nécessité de la détection précoce des signaux une fois le médicament commercialisé.

Les différentes méthodes d'étude de la disproportionnalité des notifications incluent des méthodes fréquentistes, telles que les études cas-non cas ou les études du proportional reporting ratio (PRR) et des méthodes bayésiennes telles que la méthode multi-item gamma Poisson Shrinker (MGPS) [1] utilisée plus spécifiquement par la FDA ou la méthode Bayesian confidence propagation neural network (BCPNN) [2] utilisée par l'OMS.

\section{Principe de l'étude cas-non cas}

Afin de mettre en évidence un lien entre la notification de l'effet indésirable d'intérêt et le médicament d'intérêt, le principe de l'étude cas-non cas repose sur l'analyse d'un tableau de contingence à double entrée décrivant le nombre de notifications dans la base de données selon la notification de l'effet indésirable d'intérêt et la mention dans la 


\begin{tabular}{|l|c|c|}
\hline Exposition EIM & $\begin{array}{c}\text { EIM d'intérêt } \\
\text { « Cas » }\end{array}$ & $\begin{array}{c}\text { Autres EIM } \\
\text { « Non cas » }\end{array}$ \\
\hline Médicament d'intérêt & $a$ & $b$ \\
\hline Autres médicaments & $c$ & $d$ \\
\hline
\end{tabular}

Figure 1. Tableau de contingence pour l'analyse cas-non cas. EIM : effet indésirable médicamenteux.

notification traduisant l'exposition au médicament d'intérêt (Fig. 1).

Les notifications de l'effet indésirable d'intérêt sont appelées «cas » et les autres notifications sont appelées «non cas». Malgré ses similitudes avec la méthode cas-témoins, on n'emploie pas le terme de «témoins » mais de «non cas» car ce groupe fait référence à des patients qui ont tous été exposés à au moins un médicament et ont tous présenté au moins un événement autre que l'effet indésirable d'intérêt. Classiquement, le nombre de notifications dans chacune des cases du tableau de contingence est représenté avec les lettres $a, b$, c et $d$, correspondant respectivement aux cas exposés au médicament d'intérêt, aux non cas exposés au médicament d'intérêt, aux cas exposés aux autres médicaments et aux non cas exposés aux autres médicaments. En l'absence de signal, la distribution des cas et des non cas est indépendante de l'exposition au médicament d'intérêt, c'est-à-dire similaire entre les notifications exposées au médicament d'intérêt et celles exposées aux autres médicaments. Un signal est mis en évidence lorsque l'on démontre une différence de cette distribution statistique (une «disproportionnalité »).

La première fois que la méthode cas-non cas a été utilisée remonte à 1982 lors d'une étude explorant le lien entre l'utilisation d'acide valproïque pendant la grossesse et les malformations fœtales de type spina bifida, à partir d'un registre de malformations fœtales de la région de Lyon en France [3]. Parmi les 146 mères dont l'enfant présentait un spina bifida, 9 avaient été exposées à l'acide valproïque au premier trimestre de la grossesse et parmi les 6616 mères d'enfant présentant d'autres malformations, 21 avaient été exposées, ce qui donnait un rapport de cotes de 20,6 $(p<0,001)$. Les auteurs ont donc utilisé un design de type cas-témoin dans lequel les témoins étaient des cas de malformations congénitales autres que le spina bifida, autrement dit des «non cas ». Cette étude a ainsi été rétrospectivement considérée comme la première étude cas-non cas [4].

La démonstration de la disproportionnalité repose sur le calcul d'un rapport de cotes de notification, appelé reporting odds ratio (ROR) [5,6]. Le terme et le calcul du ROR a été présenté pour la première fois par Stricker et Tijssen en 1992, lors de l'étude du lien entre maladie sérique et céfaclor (par rapport à d'autres antibiotiques) dans une base de données de pharmacovigilance internationale [7]. Emprunté au calcul de l'odds ratio des études cas-témoins, le calcul du ROR repose sur une méthode simple. Pour un groupe d'exposition, la cote de notification est égale à la fréquence de notification (f) divisée par (1-f). Ainsi, pour le groupe exposé au médicament d'intérêt, la fréquence de notification correspond à $a /(a+b)$ et la cote de notification est égale à $[a /(a+b)] /[1-a /(a+b)]$ qui se simplifie en $a / b$. De la même manière, pour le groupe exposé aux autres médicaments, la cote de notification est ainsi égale à $c / d$. Le ROR peut être généralement calculé si aucune case du tableau de contingence ne présente de valeur nulle [6]. Il correspond au rapport des cotes de notification entre les groupes exposés ou non au médicament d'intérêt, et vaut donc $(\mathrm{a} / \mathrm{b}) /(\mathrm{c} / \mathrm{d})$, soit ad/bc [8]. Le ROR mesure la force de la disproportionnalité, si le ROR vaut 1 , il n'y a pas de signal : l'effet indésirable d'intérêt est autant notifié avec le médicament d'intérêt qu'avec les autres médicaments (si le ROR est inférieur à 1, il n'y a fortiori pas non plus de signal, l'effet indésirable étant moins notifié avec le médicament d'intérêt qu'avec les autres médicaments). En revanche, si le ROR est supérieur à 1 , les cas sont plus notifiés avec le médicament d'intérêt qu'avec les autres médicaments, c'est un signal et plus la valeur du ROR est élevée, plus cette disproportionnalité est grande. Toutefois, le ROR est une estimation statistique et il doit toujours être présenté et interprété avec son intervalle de confiance à $95 \%$ (IC $95 \%)$. Le calcul de l'IC $95 \%$ du ROR fait intervenir la variance du logarithme népérien du ROR qui s'exprime de manière simplifiée par la formule :

$1 / a+1 / b+1 / c+1 / d$

Ainsi, l'IC $95 \%$ du ROR peut se calculer de la manière suivante :

$R O R * e^{+1.96 \sqrt{\frac{1}{a}+\frac{1}{b}+\frac{1}{c}+\frac{1}{d}}}$

Si l'IC $95 \%$ du ROR est dans son intégralité supérieure à la valeur 1 (i.e. la borne inférieure de l'IC $95 \%$ est supérieure à 1), alors le signal de disproportionnalité est statistiquement significatif et on interprète généralement le ROR de la manière suivante : l'effet indésirable d'intérêt est ROR fois plus notifié avec le médicament d'intérêt qu'avec les autres médicaments. À l'inverse si la borne inférieure de l'IC $95 \%$ est inférieure à 1 , alors il n'y a pas de signal mis en évidence. En utilisant un modèle de régression logistique multivarié, le calcul du ROR pourra être ajusté sur des cofacteurs pouvant jouer un effet de confusion (âge, sexe, antécédents, comédications, notificateur, région, etc.). Dans l'étude présentée par Stricker et Tijssen, le ROR pour les notifications de maladie sérique avec le céfaclor en comparaison à l'amoxicilline était de 12,4 (IC $95 \%$ : 8,2-18,7), montrant ainsi un signal largement statistiquement significatif : les notifications de maladie sérique étaient environ 12 fois plus notifiées avec le céfaclor qu'avec l'amoxicilline [7]. Ce ROR était ajusté sur le pays de notification, l'âge, le sexe, l'origine et l'année de notification.

\section{Comparaison aux autres méthodes d'étude de la disproportionnalité}

Parmi les autres mesures de disproportionnalité, le proportional reporting ratio (PRR) se calcule également à partir du tableau de contingence et vaut $[a /(a+b)] /[c /(c+d)]$. On considère généralement qu'un signal est présent lorsque le PPR est supérieur à 2, la valeur de la statistique du Chi ${ }^{2}$ supérieure à 4 et le nombre de cas est supérieur à 3 [5]. La valeur du PPR est généralement similaire à celle du ROR si l'effet indésirable et l'exposition au médicament d'intérêt 
sont rares dans la base de données. Concernant les méthodes bayésiennes, par exemple pour la méthode Bayesian confidence propagation neural network (BCPNN), la mesure de disproportionnalité s'exprime sous la forme de l'information component (IC) qui s'interprète par rapport à la valeur zéro. Bien que les méthodes bayésiennes soient considérées comme plus robustes que les méthodes fréquentistes quand le nombre de cas exposés est faible, Chen et al. concluaient, à partir d'une étude comparant les différentes mesures de disproportionnalité sur 10 combinaisons médicament-effet indésirable dans la base de données de notifications de la FDA, que le ROR présenterait une meilleure performance que les autres techniques en termes de précocité du signal [9]. Un autre avantage du ROR est qu'il permet l'utilisation d'une régression logistique multivariée et donc la prise en compte des effets de confusion et d'interaction [6].

\section{Modalités de l'analyse de disproportionnalité}

L'étude de la disproportionnalité peut être réalisée selon diverses modalités méthodologiques. En premier lieu, des choix peuvent être faits concernant la temporalité des notifications. L'analyse pourra uniquement concerner une période précise (par exemple, la période totale de commercialisation du médicament d'intérêt ou la période précédant une alerte de sécurité). Les ROR pourront également être calculés en fonction du temps (ROR cumulé ou par unité de temps) pour explorer les biais relatifs au temps (cf. section sur les biais de temporalité). Deuxièmement, l'analyse pourra porter sur une sélection de notifications présentes dans la base de données. Cette sélection pourra concerner uniquement les cas graves, les cas notifiés par des professionnels de santé, les cas dont l'imputabilité du médicament d'intérêt est la plus forte (médicament jugé « suspect »), les effets indésirables correspondant à une spécialité médicale précise (ex. dermatologie, psychiatrie, etc.), ou les effets indésirables concernant des médicaments d'une classe thérapeutique spécifique (ex. médicaments du diabète, antibiotiques, etc.). Des exclusions de notifications permettront également d'explorer des bais de compétition (cf. section sur les biais de compétition). L'analyse de disproportionnalité sur une combinaison médicament-effet indésirable peut également être complétée par l'analyse des autres effets indésirables associés à l'évènement d'intérêt ou par l'analyse des médicaments de la même classe pharmacologique ou thérapeutique que le médicament d'intérêt. Toutes ces modalités peuvent faire l'objet d'analyses secondaires ou de sensibilité explorant la robustesse des résultats principaux.

\section{Avantages et limites des études cas-non cas}

Les principaux avantages des études de disproportionnalité sont, d'une part, qu'elles permettent l'étude d'effets indésirables rares voire très rares, il ne suffit que de quelques notifications de l'effet d'intérêt pour réaliser l'analyse. D'autre part, en comparaison aux essais cliniques, elles concernent en théorie la population exhaustive des utilisateurs (sous-groupes spécifiques compris : personnes âgées, enfants, patients avec comorbidités, etc.) et représente donc l'utilisation du médicament en condition réelle. Aussi, ce type d'étude permet la détection de nouveaux signaux de manière précoce et pouvant être automatisée, ce qui permet de justifier ultérieurement la réalisation d'études observationnelles confirmatoires. À de nombreuses reprises, les études cas-non cas ont montré l'intérêt d'une telle méthodologie dans la surveillance du risque médicamenteux. Par exemple, une étude cas-non cas des notifications de cancer de la vessie associés à la pioglitazone dans la base de données de pharmacovigilance de la FDA entre 2004 et 2009 révélait qu'un signal de disproportionnalité était présent dès l'année 2004 (ROR 4,8, IC $95 \%$ : 1,3-15,9) [10], c'est-à-dire 7 ans avant que le risque ne soit confirmé et que des mesures réglementaires ne soient mises en place [11]. Toutefois, les études confirmatoires ne sont pas toujours réalisables et, quand elles le sont, ne permettent pas toujours de conclure. Par exemple, le signal de pancréatites aiguës associées aux incrétinomimétiques suggéré par plusieurs études cas-non cas dans différentes bases de données de pharmacovigilance [12-14] n'a pour l'instant pas pu être confirmé par des études pharmaco-épidémiologiques, laissant le risque à l'état de signal. La logistique des études cas-non cas est relativement simple, leur coût est faible et le développement des standards internationaux ont permis la constitution de bases de données de pharmacovigilance accessibles pour l'étude d'effets au niveau international.

La méthode cas-non cas permet aussi d'utiliser les données pharmacodynamiques pour explorer des hypothèses mécanistiques du risque iatrogène. Par exemple, une étude s'intéressant à l'inhibition des canaux potassiques de type human ether-a-go-go-related gene (HERG) par des médicaments a utilisé une méthode cas-non cas pour montrer que l'activité anti-HERG des médicaments était directement associée à des notifications plus fréquentes d'arythmies ventriculaires graves et de mort subite dans la base de données de pharmacovigilance l'OMS. Les auteurs préconisaient ainsi la mesure de l'activité anti-HERG lors des tests précliniques des médicaments pour prédire leur effet proarythmique [15]. Un autre exemple est celui d'une étude cas-non cas combinant les données pharmacodynamiques d'activité des antipsychotiques sur les récepteurs sérotoninergiques, histaminiques, muscariniques, adrénergiques ou dopaminergiques, aux données de notifications d'effets indésirables associés aux antipsychotiques, afin d'identifier les profils pharmacodynamiques à risque. Ainsi, il était montré que l'occupation des récepteurs sérotoninergiques 5-HT2c et histaminiques H1 était associés à une notification accrue de cas de diabète sucré [16]. L'étude de disproportionnalité peut aussi être appliquée à d'autres sources de données comme des enquêtes pharmaco-épidémiologiques. Par exemple, dans le domaine de l'addictovigilance, le potentiel d'abus des médicaments psychoactifs a été étudié en comparant les estimateurs de disproportionnalité de critères tels que l'abus et la dépendance, l'acquisition illégale, la voie d'administration détournée et la consommation d'alcool concomitante à partir de données issues d'enquêtes transversales annuelles sur les consommations de produits psychoactifs par les patients abuseurs ou addicts [17]. 
Concernant les limites des études cas-non cas, la sousnotification des effets indésirables médicamenteux est une limite importante à l'interprétation de leurs résultats. Elle est inhérente au système de pharmacovigilance qui n'a pas pour objectif d'atteindre l'exhaustivité des effets indésirables mais qui se focalise sur leur pertinence pour alerter sur un risque. Ainsi seulement $5 \%$ à $10 \%$ des effets indésirables médicamenteux seraient notifiés [18]. Cette sous-notification compromet la mesure de l'incidence réelle de l'iatrogénie médicamenteuse et limite la sensibilité de la détection de signaux par cette méthode. De plus, la sous-notification est variable (on parle de « déclaration sélective »), ce taux étant influencé par de nombreux paramètres dépendants du notificateur, du médicament, de l'effet lui-même ou du temps. Ainsi, un signal de disproportionnalité peut être la conséquence soit d'une augmentation réelle de l'incidence de l'effet indésirable, soit d'une augmentation des notifications sans augmentation de l'incidence engendrant ainsi un problème de spécificité de la détection du signal. Les facteurs de variation de la notification incluent l'expérience et type de notificateur, les habitudes locales d'utilisation médicamenteuse et de notification, la gravité de l'effet indésirable (les effets indésirables graves étant davantage notifiés), la spécificité de l'effet indésirable, son caractère inattendu par rapport au profil de sécurité connu du médicament, au terrain ou à la pathologie (les effets indésirables inattendus étant davantage notifiés [19]), la médiatisation d'un effet indésirable (effet de notoriété) ou le délai depuis l'autorisation de mise sur le marché (effet Weber). Ces facteurs constituent une partie des principaux biais limitant l'interprétation des études de disproportionnalité.

Le Tableau 1 présente les biais et limites des études cas-non cas, leur conséquence et les méthodes permettant de les mesurer ou de les éviter.

\section{Biais des études de disproportionnalité}

\section{Biais de temporalité}

L'effet Weber est un phénomène de variation de la notification dans le temps qui se traduit par l'augmentation des notifications après la commercialisation d'un médicament (du fait du profil de sécurité mal connu et de l'exposition croissante), en général pendant les deux premières années, avec une grande part d'effets non graves et suivie d'un déclin (diminution de l'enthousiasme pour la notification d'effet indésirables devenant mieux connus) $[20,21]$. L'effet Weber peut s'observer également pour un médicament ancien bénéficiant d'une nouvelle indication ou d'un changement de posologie. L'impact de l'effet Weber sur l'estimation du ROR est variable et dépend de son degré d'intensité sur l'effet indésirable d'intérêt (augmentation du nombre [a]) par rapport aux autres effets indésirables (augmentation du nombre [b]). Dans tous les cas, les analyses de disproportionnalité seront moins efficaces pour détecter les effets à long délai d'apparition car à distance de la mise sur le marché. Pour les effets graves, l'effet Weber comportant une grande part d'effets non graves peut constituer un effet masquant par une augmentation plus importante des non cas exposés (nombre [b]), potentiellement responsable d'une sous-estimation du ROR. Cet effet peut donc justifier de restreindre l'analyse cas-non cas aux effets indésirables graves uniquement.

L'effet de notoriété a pour conséquence une augmentation des notifications après médiatisation d'un effet indésirable associé à un médicament (publication d'une étude scientifique, alerte de sécurité des agences réglementaires destinée aux professionnels de santé ou au public, article ou discussion dans les médias publics ou les réseaux sociaux) [22]. On observe ainsi une augmentation des notifications de cas incidents, mais aussi des cas rétrospectifs (étant survenus avant la médiatisation). Dans tous les cas, cet effet entraîne un biais responsable d'une augmentation du ROR pour le médicament en cause. Pour l'éviter, il faudra, si cela est possible, s'assurer de la présence du signal avant l'évènement médiatique. Pariente et al. ont donné plusieurs illustrations de ce biais. Par exemple, les ROR relatifs aux accidents vasculaires cérébraux (AVC) survenus sous neuroleptiques atypiques dans la base de données nationale de pharmacovigilance française étaient de 0,1 (IC $95 \%: 0,02-1,0)$ et 2,0 (IC $95 \%: 1,2-3,4)$ respectivement avant et après la publication d'un communiqué de presse de l'Agence française de sécurité sanitaire des produits de santé faisant état d'une augmentation de ce risque dans un essai clinique chez un population de personnes âgées atteinte de démence [22]. Dans les situations de notoriété d'un effet indésirable avec un médicament, il est également possible d'observer un effet de ricochet (ripple effect) se traduisant par une augmentation des notifications de l'effet étudié avec d'autres médicaments appartenant à la même classe pharmacologique ou thérapeutique que le médicament initialement suspecté dans l'alerte [22]. Il a par exemple été observé, dans la base de données de pharmacovigilance états-unienne, une augmentation de près de $40 \%$ des notifications de pancréatites avec la sitagliptine après une alerte de la FDA concernant le risque de pancréatite avec l'exenatide [13].

\section{Biais d'information}

Les données manquantes sont fréquentes dans les bases de données de pharmacovigilance, toutefois, elles ne concernent généralement pas l'identification du médicament ni le diagnostic de l'effet et n'empêchent donc pas la réalisation de l'analyse de disproportionnalité en elle-même. En revanche, des renseignements manquants sur les données cliniques, l'indication, l'âge, le sexe, les comédications, l'évolution, le type de notification et de notificateur, le pays, les doses et dates précises du traitement limitent les analyses complémentaires et les ajustements sur des facteurs de confusion potentiels. Sur la totalité des notifications présentes dans VigiBase, le sexe et l'âge sont manquants dans $6,0 \%$ et $25,6 \%$ des cas, respectivement [23]. Un score de complétude des notifications, appelé VigiGrade, a été développé par le centre d'Uppsala gestionnaire de VigiBase. Son calcul est réalisé en attribuant des pénalités selon la disponibilité des informations et leur pertinence clinique [24]. On considère qu'une notification est bien documentée lorsque le score VigiGrade est supérieur à 0,8. En 2014, seulement $13 \%$ des notifications présentes dans VigiBase correspondaient à ce critère et ce taux présente une grande variabilité selon le pays (par exemple, il est 
Tableau 1 Principaux biais et limites des études cas-non cas, leurs conséquences et, le cas échéant, les méthodes permettant de les mesurer ou de les éviter.

\begin{tabular}{|c|c|c|}
\hline Biais/limite & Conséquences & Solutions \\
\hline Sous-notification & $\begin{array}{l}\text { Absence de mesure de l'incidence réelle et } \\
\text { du risque par rapport aux non exposés } \\
\text { Manque de puissance statistique } \\
\text { Diminution de la sensibilité de détection de } \\
\text { signal }\end{array}$ & \\
\hline Déclaration sélective & ROR sur- ou sous-estimé & $\begin{array}{l}\text { Restriction ou ajustement sur des facteurs } \\
\text { influençant la notification (gravité, type de } \\
\text { notificateur, région, ...) } \\
\text { Témoins positifs et négatifs explorant la } \\
\text { notification de l'effet étudié }\end{array}$ \\
\hline Effet Weber & Variabilité du ROR dans le temps & Analyse temporelle du ROR \\
\hline & $\begin{array}{l}\text { ROR surestimé ou sous-estimé si effet } \\
\text { masquant par des cas non graves }\end{array}$ & Restriction aux cas graves si effet masquant \\
\hline $\begin{array}{l}\text { Biais de notoriété et } \\
\text { effet de ricochet }\end{array}$ & ROR surestimé & $\begin{array}{l}\text { Analyse temporelle du ROR } \\
\text { Restriction de l'analyse dans le temps } \\
\text { (période précédent une médiatisation) }\end{array}$ \\
\hline Données manquantes & $\begin{array}{l}\text { Contrainte pour les analyses } \\
\text { complémentaires et les ajustements sur les } \\
\text { cofacteurs }\end{array}$ & $\begin{array}{l}\text { Comparer l'analyse restreinte aux données } \\
\text { renseignées et celle avec création d'une } \\
\text { catégorie « donnée manquante » } \\
\text { Exploration des possibilités d'imputation }\end{array}$ \\
\hline $\begin{array}{l}\text { Notifications multiples } \\
\text { pour un même cas }\end{array}$ & ROR erroné & « Dédoublonnage » manuel ou algorithmique \\
\hline $\begin{array}{l}\text { Biais d'information sur } \\
\text { les données cliniques } \\
\text { et d'exposition }\end{array}$ & ROR erroné & $\begin{array}{l}\text { Restriction aux notifications médicalement } \\
\text { confirmées } \\
\text { Restriction de l'exposition aux notifications } \\
\text { pour lesquelles le médicament est } \\
\text { jugé « suspect » } \\
\text { Attention portée aux définitions à partir des } \\
\text { classifications de pathologies et de } \\
\text { médicaments, et utilisation de standards } \\
\text { (SMQ) }\end{array}$ \\
\hline $\begin{array}{l}\text { Biais de sélection et } \\
\text { choix du groupe de } \\
\text { référence }\end{array}$ & $\begin{array}{l}\text { ROR erroné } \\
\text { Mauvaise spécificité }\end{array}$ & $\begin{array}{l}\text { Groupe de référence constitué des } \\
\text { médicaments de la même classe } \\
\text { pharmacologique ou thérapeutique }\end{array}$ \\
\hline Biais de confusion & ROR erroné & $\begin{array}{l}\text { Stratification ou ajustement sur les } \\
\text { cofacteurs influençant la notification et les } \\
\text { facteurs de risque de l'effet }\end{array}$ \\
\hline $\begin{array}{l}\text { Biais de compétition } \\
\text { entre médicaments }\end{array}$ & ROR sous-estimé & $\begin{array}{l}\text { Exclusion des notifications concernant les } \\
\text { médicaments fréquemment associés à } \\
\text { l'effet indésirable d'intérêt }\end{array}$ \\
\hline $\begin{array}{l}\text { Biais de compétition } \\
\text { entre événements }\end{array}$ & ROR sous-estimé & $\begin{array}{l}\text { Exclusion des notifications concernant les } \\
\text { effets indésirables connus pour être } \\
\text { fréquemment associés au médicament } \\
\text { d'intérêt }\end{array}$ \\
\hline Biais protopathique? & ROR erroné & $\begin{array}{l}\text { Observation de période de latence suivant } \\
\text { l'exposition }\end{array}$ \\
\hline $\begin{array}{l}\text { Biais de surveillance ou } \\
\text { de détection? }\end{array}$ & ROR surestimé & $\begin{array}{l}\text { Recherche de comparateur avec profil de } \\
\text { surveillance similaire }\end{array}$ \\
\hline Tous biais & ROR erroné & $\begin{array}{l}\text { Exploration des biais en réalisant des } \\
\text { analyses de sensibilité } \\
\text { Recherche de résultats concordants pour } \\
\text { des témoins positifs et négatifs }\end{array}$ \\
\hline
\end{tabular}

ROR : reporting odds ratio ; SMQ : standardised MedRA queries. 
globalement meilleur en Europe, qu'aux États-Unis), le type de notificateur (24\% pour les médecins contre $4 \%$ pour les consommateurs/non professionnels de santé) [24], mais aussi selon le contexte clinique (par exemple, il y a plus de données manquantes pour les cas graves chez les diabétiques) ou le circuit des notifications (plus de données manquantes pour les cas notifiés par les industriels) [25]. En 2016, la France figurait parmi les 10 pays les plus contributifs à VigiBase [26] avec un score de complétude médian égal à 0,80 [27]. Il n'existe pas de solution idéale pour prendre en compte les données manquantes mais, outre les possibilités d'imputation, il peut être utile pour explorer ce biais de comparer les analyses restreintes aux données renseignées et celles avec création d'une catégorie «donnée manquante ». Les notifications multiples d'un même effet indésirable sont une source d'erreur importante et le «dédoublonnage » est la première étape de toute analyse utilisant les bases de données de pharmacovigilance. VigiBase utilise désormais un algorithme permettant de repérer et de supprimer les doublons (VigiMatch) en gardant les notifications les plus informatives. De manière similaire, du fait de l'anonymisation des notifications et le manque d'identifiant unique par patient, il est souvent difficile de réaliser une analyse avec le patient comme unité statistique, dans ce cas, les événements multiples pour un même patient (par exemple, un effet récurent) comptent comme plusieurs observations indépendantes.

Les erreurs dans les notifications sur le diagnostic, l'exposition ou l'imputation sont souvent dépendantes du type de notificateur. Afin de limiter ce biais d'information, il est possible de sélectionner les observations en limitant l'analyse aux observations pour lesquelles le risque d'erreur est plus faible : les effets indésirables notifiés par des professionnels de santé, les notifications dans lesquelles le médicament est jugé « suspect » ou présentant une chronologie suggestive. Pour éviter les erreurs sur l'exposition, il faut porter une attention particulière à la définition de l'exposition à partir des classifications médicamenteuses (une erreur peut par exemple être secondaire à la non prise en compte des codes anatomique, thérapeutique et chimique [ATC] des formes associées du médicament à l'étude). Pour définir l'effet étudié, la sélection des termes rapportés à partir des classifications d'effets d'indésirables peut être de pertinence clinique incertaine et il est recommandé d'utiliser, lorsque cela est possible, les standardised MedDRA queries (SMQ) qui proposent des sélections de termes cliniques avec des définitions «narrow » (plus spécifique) ou «broad» (plus sensible). Aussi, la recherche de données cliniques (indication, antécédents) à partir de proxy basés sur les expositions médicamenteuses peut également être une source de biais d'information. Les analyses temporelles peuvent être également limitées par les délais entre la survenue de l'effet et enregistrement des notifications dans les bases de données, a fortiori internationales. Par exemple, dans VigiBase, certains pays enregistrent les notifications chaque mois, d'autres chaque trimestre ou deux fois par an.

\section{Biais de sélection ou de confusion}

Dans les analyses de disproportionnalité, comme dans les études de pharmaco-épidémiologie en général, les comparaisons peuvent être biaisées par le fait que les patients exposés au médicament d'intérêt peuvent être plus (ou moins) à risque pour l'effet indésirable que ceux exposées aux autres médicaments (biais d'indication, biais de sévérité, channeling bias ou healthy user effect). Un «surrisque » dans le groupe exposé se traduira par une «surnotification » et donc un faux signal. Pour limiter ces effets, le choix du groupe de référence est crucial. Choisir tous les " autres médicaments » comme groupe de référence peut être problématique en présence d'un biais d'indication : le signal reflèterait le risque associé à l'indication et non au médicament lui-même. Par exemple, pour l'étude des notifications de pancréatite associées à l'exenatide indiquée pour le traitement du diabète de type 2 , si le groupe de référence inclus tous les autres médicaments et pas seulement les médicaments du diabète, le signal observé pourra être expliqué par l'effet de l'indication (le diabète étant un facteur de risque de pancréatite). Le choix du groupe de référence devra ainsi se porter sur les autres médicaments de la même classe pharmacologique ou thérapeutique (dans cet exemple, les médicaments du diabète). Une étude réalisée dans la base de données EudraVigilance a comparé les résultats d'analyses de disproportionnalité (en utilisant des PRR) sans et avec restriction du groupe de référence à la classe thérapeutique des médicaments étudiés (médicaments des troubles prostatiques et médicaments du diabète de type 2). Les auteurs démontraient qu'en utilisant ce type de restriction, la performance de l'estimateur de disproportionnalité était améliorée avec une diminution des faux positifs (confondus par la maladie) et une meilleure capacité à détecter les vrais positifs [28]. Aussi, lors d'une étude pilote évaluant un score de hiérarchisation des signaux issus de l'analyse des bases de pharmacovigilance, Salvo et al. préconisaient d'ajouter des critères pharmacologiques incluant notamment le ROR calculé parmi la classe thérapeutique du médicament étudié afin d'augmenter la spécificité du signal [29].

Les facteurs de confusion dans les analyses de disproportionnalité peuvent être des facteurs confondant la relation entre l'exposition et la notification de l'effet (potentiellement tous les facteurs responsables de la déclaration sélective présentés plus haut) mais aussi ceux, plus classiques, confondant la relation entre l'exposition et l'effet lui-même. Par exemple, un signal concernant le risque d'hypoglycémie associé aux inhibiteurs de l'enzyme de conversion (IEC) était identifié dans la base de données nationale de pharmacovigilance française (ROR entre 3 et 4) mais en réalité, le diabète était un facteur de confusion : les IEC sont plus favorablement prescrits aux patients diabétiques par ailleurs traités par antihyperglycémiants favorisant les épisodes d'hypoglycémies et donc leur notification. Lors de l'analyse stratifiée sur la présence ou non d'antihyperglycémiants, le signal disparaissait (ROR à 1 dans chaque strate) [30].

\section{Biais de compétition}

Il peut exister un biais de compétition entre médicaments lorsque l'effet indésirable étudié est également fréquemment notifié avec un ou plusieurs autre(s) médicament(s) que le médicament d'intérêt [31]. Ainsi, on observe une importante déclaration de cas non exposés (nombre [c] du 
tableau de contingence), ce qui peut entraîner une sousestimation du ROR c'est-à-dire un signal passant inaperçu. Ce biais augmente les faux négatifs et diminue donc la sensibilité de la détection du signal. La solution proposée est de retirer de l'analyse les notifications concernant les médicaments fréquemment associés à l'effet indésirable d'intérêt : ceci permet de diminuer le nombre de cas exposés nécessaires pour montrer un signal. Dans l'exemple des AVC survenus sous neuroleptiques atypiques, il existe un biais de compétition entre antipsychotiques et anticoagulants, ces derniers étant connus pour augmenter le risque d'AVC et donc leur notification. L'exclusion des notifications concernant anticoagulants révélait un signal d'AVC avec les neuroleptiques atypiques qui passait auparavant inaperçu : ROR à 1,1 (IC $95 \%: 0,7-1,9$ ) et 2,0 (IC $95 \%: 1,2-3,4$ ) respectivement avant et après l'exclusion [31].

On peut observer un biais de compétition entre événements lorsque le médicament d'intérêt est associé à une importante déclaration d'effet(s) indésirable(s) autre(s) que l'effet indésirable d'intérêt [32]. Il existe alors une importante déclaration de non cas exposés (nombre [b] du tableau de contingence), ce qui peut entraîner une sous-estimation du ROR et une baisse de la sensibilité. La solution est similaire au cas précédent : on retire de l'analyse les notifications d'effets indésirables connus pour être fréquemment associés au médicament d'intérêt. Par exemple, dans une étude de disproportionnalité sur les statines, le retrait des notifications d'affections musculaires, un effet indésirable connu des statines, permet de révéler des signaux auparavant inaperçus : trouble de la libido, alopécie, cholestase, dermatites, hémorragies digestive, etc [32]. Cet effet justifie également le fait de réaliser l'analyse de disproportionnalité restreinte aux notifications appartenant au système-organe-classe spécifique de l'effet indésirable d'intérêt (par exemple dermatologique, psychiatrique, etc.).

\section{Autres biais potentiels}

D'autres biais potentiels souvent évoqués dans les études pharmaco-épidémiologiques pourraient également limiter l'interprétation des études de disproportionnalité. On peut citer par exemple le biais protopathique qui survient lorsque qu'un médicament est initié en réponse à un symptôme ou un stade précoce de l'effet suspecté. Par exemple, l'utilisation d'analgésiques en réponse à la douleur causée par une tumeur non diagnostiquée peut conduire, lorsque la tumeur est diagnostiquée, à des notifications suggérant que l'analgésique a causé la tumeur alors que la causalité est inversée. Dans cette situation, il faut rétablir la chronologie en introduisant une période de latence après l'exposition pendant laquelle on considère les cas survenus comme non exposés. Le biais de surveillance ou biais de détection est un autre exemple à garder à l'esprit. Il survient lorsque la détection de l'évènement est plus probable dans un groupe d'exposition en raison d'une surveillance accrue, d'un dépistage de la maladie ou d'un symptôme associé. Par exemple, des notifications suggérant un risque de cancer de l'endomètre avec les traitements hormonaux substitutifs (THS) pourraient théoriquement être la conséquence d'une meilleure détection de ces cancers chez les femmes traités par THS. En effet, les THS sont connus pour provoquer des saignements utérins qui sont des symptômes motivant le dépistage du cancer de l'endomètre.

\section{Témoins positifs et négatifs pour évaluer la présence de biais}

La présence de biais non détectés peut être recherchée en étudiant des médicaments témoins dont l'association ou l'absence d'association avec l'effet étudié est attendue selon les données de la littérature. Il s'agit de mesurer la disproportionnalité :

- de médicaments connus pour entraîner l'effet indésirable (témoins positifs) ;

- de médicaments n'ayant aucun lien avec l'effet indésirable (témoins négatifs) et de s'assurer que l'on retrouve un signal pour les témoins positifs et aucun signal pour les témoins négatifs.

La cohérence des résultats obtenus avec les résultats attendus pour les témoins suggèrerait que l'étude n'est pas compromise par des biais majeurs. Dans l'étude du lien entre antihypertenseurs et hypoglycémie, Grégoire et al. ont utilisé la cibenzoline et le disopyramide comme témoins positifs et le diazépam comme témoin négatif. Comme attendu, un signal pour le risque d'hypoglycémie était retrouvé pour la cibenzoline (ROR 107 ; IC $95 \%$ : 78-148) et le disopyramide (ROR 17 ; IC $95 \%$ : 10-29) alors qu'aucun signal n'était mis en évidence pour le diazépam (ROR 0,3 ; IC $95 \%: 0,1-2$ ) [30].

\section{Conclusions}

Les études cas-non cas comme les autres études de disproportionnalité analysent les données de surveillance de la vie réelle et, malgré d'importantes limites et de nombreux biais, permettent la détection de signaux de pharmacovigilance utiles pour planifier la réalisation d'études confirmatoires (souvent pharmaco-épidémiologiques). Les signaux issus des études cas-non cas, couplés à l'analyse clinique et pharmacologique, restent des éléments importants du faisceau d'arguments nécessaire pour mettre en évidence les risques médicamenteux et sont parfois les seuls outils à disposition des pharmacovigilants en particulier pour l'étude des événements rares ou des médicaments faiblement utilisés [33].

\section{Déclaration de liens d'intérêts}

L'auteur déclare ne pas avoir de liens d'intérêts.

\section{Références}

[1] DuMouchel W. Bayesian data mining in large frequency tables, with an application to the fda spontaneous reporting system. Am Stat 1999;53:177.

[2] Bate A, Lindquist M, Edwards IR, Olsson S, Orre R, Lansner A, et al. A Bayesian neural network method for adverse drug reaction signal generation. Eur J Clin Pharmacol 1998;54:315-21.

[3] Bjerkedal T, Czeizel A, Goujard J, et al. Valproic acid and spina bifida. Lancet Lond Engl 1982;2(8307):1096. 
[4] Montastruc JL, Sommet A, Bagheri H, Lapeyre-Mestre M. Benefits and strengths of the disproportionality analysis for identification of adverse drug reactions in a pharmacovigilance database. Br J Clin Pharmacol 2011;72:905-8.

[5] Moore N, Thiessard F, Begaud B. The history of disproportionality measures (reporting odds ratio, proportional reporting rates) in spontaneous reporting of adverse drug reactions. Pharmacoepidemiol Drug Saf 2005;14:285-6.

[6] van Puijenbroek EP, Bate A, Leufkens HGM, Lindquist M, Orre R, Egberts ACG. A comparison of measures of disproportionality for signal detection in spontaneous reporting systems for adverse drug reactions. Pharmacoepidemiol Drug Saf 2002;11:3-10.

[7] Stricker BH, Tijssen JG. Serum sickness-like reactions to cefaclor. J Clin Epidemiol 1992;45:1177-84.

[8] Moore N, Kreft-Jais C, Haramburu F, Noblet C, Andrejak M, Ollagnier $M$, et al. Reports of hypoglycaemia associated with the use of ACE inhibitors and other drugs: a case/non-case study in the French pharmacovigilance system database. $\mathrm{Br} \mathrm{J}$ Clin Pharmacol 1997;44:513-8.

[9] Chen Y, Guo JJ, Steinbuch M, Lin X, Buncher CR, Patel NC. Comparison of sensitivity and timing of early signal detection of four frequently used signal detection methods: an empirical study based on the US FDA Adverse Event Reporting System Database. Pharm Med 2008;22:359-65.

[10] Piccinni C, Motola D, Marchesini G, Poluzzi E. Assessing the association of pioglitazone use and bladder cancer through drug adverse event reporting. Diabetes Care 2011;34:1369-71.

[11] Faillie JL, Petit P, Montastruc JL, Hillaire-Buys D. Scientific evidence and controversies about pioglitazone and bladder cancer: which lessons can be drawn? Drug Saf 2013;36:693-707

[12] Elashoff M, Matveyenko AV, Gier B, Elashoff R, Butler PC. Pancreatitis, pancreatic, and thyroid cancer with glucagon-like peptide-1-based therapies. Gastroenterology 2011;141:150-6.

[13] Raschi E, Piccinni C, Poluzzi E, Marchesini G, De Ponti F. The association of pancreatitis with antidiabetic drug use: gaining insight through the FDA pharmacovigilance database. Acta Diabetol 2013;50:569-77.

[14] Faillie JL, Babai S, Crepin S, Bres V, Laroche ML, Le Louet H, et al. Pancreatitis associated with the use of GLP-1 analogs and DPP-4 inhibitors: a case/non-case study from the French Pharmacovigilance Database. Acta Diabetol 2014;51:491-7.

[15] De Bruin ML, Pettersson M, Meyboom RHB, Hoes AW, Leufkens HGM. Anti-HERG activity and the risk of drug-induced arrhythmias and sudden death. Eur Heart J 2005;26:590-7.

[16] Montastruc F, Palmaro A, Bagheri H, Schmitt L, Montastruc $\mathrm{JL}$, Lapeyre-Mestre M. Role of serotonin 5-HT2C and histamine $\mathrm{H} 1$ receptors in antipsychotic-induced diabetes: a pharmacoepidemiological-pharmacodynamic study in VigiBase. Eur Neuropsychopharmacol 2015;25:1556-65.

[17] Pauly V, Lapeyre-Mestre M, Braunstein D, Rueter M, Thirion $\mathrm{X}$, Jouanjus $\mathrm{E}$, et al. Detection of signals of abuse and dependence applying disproportionality analysis. Eur J Clin Pharmacol 2015;71:229-36.

[18] Bégaud B, Martin K, Haramburu F, Moore N. Rates of spontaneous reporting of adverse drug reactions in France. JAMA 2002;288:1588.
[19] Martin RM, Kapoor KV, Wilton LV, Mann RD. Underreporting of suspected adverse drug reactions to newly marketed ("black triangle'”) drugs in general practice: observational study. BMJ 1998;317:119-20.

[20] Wallenstein EJ, Fife D. Temporal patterns of NSAID spontaneous adverse event reports: the Weber effect revisited. Drug Saf 2001;24:233-7.

[21] Hartnell NR, Wilson JP. Replication of the Weber effect using postmarketing adverse event reports voluntarily submitted to the United States Food and Drug Administration. Pharmacotherapy 2004;24:743-9.

[22] Pariente A, Gregoire F, Fourrier-Reglat A, Haramburu F, Moore $N$. Impact of safety alerts on measures of disproportionality in spontaneous reporting databases: the notoriety bias. Drug Saf 2007;30:891-8.

[23] Vigilyze. Search and analysis tool for VigiBaseTM the WHO global ICSR (Individual Case Safety Report) database. https://www.vigilyze.who-umc.org/ [Consulté le 25 octobre 2017].

[24] Bergvall T, Norén GN, Lindquist M. VigiGrade: a tool to identify well-documented individual case reports and highlight systematic data quality issues. Drug Saf 2014;37:65-77.

[25] Faillie JL, Robin P, Bres V, Pinzani V, Bos-Thompson MA, Hillaire-Buys D. The ATHE score: a new quality score for spontaneous adverse drug reaction reports. Fundam Clin Pharmacol 2013;27(suppl 1):106-7.

[26] Uppsala Monitoring Center (UMC). Annual Report 2015-2016. https://www.who-umc.org/media/3081/umc-annual-reportfinal-version_small.pdf [Consulté le 25 octobre 2017 (24 pp)].

[27] Vigilyze. Search and analysis tool for VigiBaseTM the WHO global ICSR (Individual Case Safety Report) database. Data from France in 2016. https://www.vigilyze.who-umc.org/\#/ [Consulté le 25 octobre 2017].

[28] Grundmark B, Holmberg L, Garmo H, Zethelius B. Reducing the noise in signal detection of adverse drug reactions by standardizing the background: a pilot study on analyses of proportional reporting ratios-by-therapeutic area. Eur J Clin Pharmacol 2014;70:627-35.

[29] Salvo F, Raschi E, Moretti U, Chiarolanza A, FourrierRéglat A, Moore N, et al. Pharmacological prioritisation of signals of disproportionate reporting: proposal of an algorithm and pilot evaluation. Eur J Clin Pharmacol 2014;70: 617-25.

[30] Grégoire F, Pariente A, Fourrier-Reglat A, Haramburu F, Bégaud B, Moore N. A signal of increased risk of hypoglycaemia with angiotensin receptor blockers caused by confounding. Br J Clin Pharmacol 2008;66:142-5.

[31] Pariente A, Didailler M, Avillach P, Miremont-Salamé G, Fourrier-Reglat A, Haramburu F, et al. A potential competition bias in the detection of safety signals from spontaneous reporting databases. Pharmacoepidemiol Drug Saf 2010;19:1166-71.

[32] Salvo F, Leborgne F, Thiessard F, Moore N, Bégaud B, Pariente A. A potential event-competition bias in safety signal detection: results from a spontaneous reporting research database in France. Drug Saf 2013;36:565-72.

[33] Faillie JL, Montastruc F, Montastruc JL, Pariente A. Pharmacoepidemiology and its input to pharmacovigilance. Therapie 2016;71:211-6. 